\title{
PRECARIZACIÓN DEL TRABAJO Y NUEVAS DESIGUALDADES: EL PAPEL DE LA INMIGRACIÓN
}

Precarization of work and new inequalities: the role of immigration

\author{
Fabio Perocco ${ }^{1}$
}

\begin{abstract}
Resumen. Este artículo se centra en el "doble papel" que puede tener la inmigración en el proceso de precarización del trabajo: por un lado interesa sobretodo y de manera considerable los trabajadores inmigrantes, por el otro, la inmigración es a menudo un vector de difusión y ampliación de precariedad. Examina el impacto de la precariedad sobre los trabajadores inmigrantes en el contexto europeo y la importancia general de las políticas migratorias en adelantar una mayor ampliación de la precariedad; después toma en consideración el contexto italiano como símbolo de la precarización del trabajo inmigrante y de la experimentación en el sector de la inmigración de nuevas formas de precariedad.
\end{abstract}

Palabras clave: precariedad, trabajadores inmigrantes, transformaciones del trabajo.

\begin{abstract}
The article focuses on the "double role" immigration may have in the process of casualization of work: on one hand such process firstly and markedly involves migrant workers, on the other hand immigration often provides a vector of spreading and increase of precarity. The article deals with the impact of precarity on migrant workers within the European context, and the global importance of migration policies in anticipating a larger expansion of precarity; then, it focuses on the Italian context as a symbol for both the precarization of migrant labour and the experimentation in the migration sector of new forms of precariousness.
\end{abstract}

Keywords: precarity, migrants workers, transformations of work.

\section{Introducción}

La generalización de la precariedad como principio cardinal de la vida de los trabajadores es una de las principales transformaciones sociales de la era neoliberal. En Europa, desde hace al menos veinte años, la flexibilidad del trabajo es uno de los temas centrales de los discursos públicos y de las agendas políticas; la nueva regulación del trabajo en nombre de la precariedad

\footnotetext{
1 Università Ca' Foscari, Venezia. Venezia, Italia.
} 
máxima y de la desestructuración de los derechos sociales ha encontrado defensores y partidarios en todas partes, pero también críticos y opositores que han subrayado sus consecuencias en cuanto a nuevas estratificaciones, nuevas desigualdades, "mal de trabajo".

La precarización del trabajo es un proceso global que se ha realizado de forma heterogénea según los contextos nacionales y que se ha concretizado en cada país de manera diferente según los sectores, las cualificaciones, las estratificaciones del mercado del trabajo; además de los jóvenes, de los older workers poco cualificados, de muchas mujeres, ha interesado principalmente y de manera muy fuerte a los inmigrantes, que han visto su precariedad laboral juntarse a la debilidad de su estado jurídico y social. La inmigración, a su pesar, también es un vector de difusión y ampliación de la precariedad, a veces es hasta un taller de experimentación de nuevas formas de precariedad que después se extienden a otras camadas de trabajadores.

Este artículo aborda este doble aspecto, el impacto de la precariedad sobre los trabajadores inmigrantes y el papel de la inmigración en su ampliación. En la primera parte, se examina el proceso de precarización estructural del trabajo, subrayando cómo este representa el corazón de la gran restructuración social de las últimas décadas y está ahora en una fase aún más extrema. La segunda parte examina la precarización laboral de los inmigrantes en Europa en las últimas dos décadas, caracterizada principalmente por la combinación de políticas migratorias, políticas de trabajo, políticas de identidad, y la significación general de estas políticas en adelantar una mayor extensión de la precariedad. La tercera parte, se enfoca en el contexto italiano como ejemplo emblemático de dicho doble proceso, examinando de un lado la precariedad extrema vivida por los trabajadores inmigrantes y del otro la experimentación en el sector de la inmigración de nuevas formas de precariedad, como voucher (bonos) y trabajo voluntario.

\section{Más allá de la precariedad: transformaciones y precarización estructural del trabajo}

Una de las piedras angulares de la gran restructuración social de las últimas tres décadas es la profunda e intensa transformación de la organización del trabajo y del mercado del trabajo ${ }^{2}$, un proceso multidimensional, pero también unitario, funcional a la mayor explotación del trabajo requerida por las reglas de la economía mundial en respuesta a la constante reducción de la tasa de acumulación y a las crisis económicas recurrentes cada vez más

\footnotetext{
2 ANTUNES, Ricardo. The Meanings of Work; DOOGAN, Kevin. New capitalism? The transformation of work; HEAD, Simon. The New Ruthless Economy; HUWS, Ursula. Labor in the Global Digital Economy; JÜRGENS, Uulrich, KRZYWDZINSKI, Martin. New Worlds of Work.
} 
cercanas y agudas. La precarización estructural del trabajo es una de las piedras angulares de estas transformaciones y ha constituido un presupuesto y un vector importante de la intensificación de la explotación del trabajo, pero la ruptura de la unidad de la relación de trabajo, la desestructuración del derecho del trabajo, la introducción de múltiples tipologías contractuales conllevantes condiciones y derechos diferentes (trabajo intermitente, compartido, a llamada, zero hours contracts, voluntario, externalizado, a licitación, formalmente autónomo, formalmente cooperativo) hacen parte de un proceso más amplio y profundo de una simple pérdida de espacios en el trabajo garantizado; después de una temporada de algunas décadas, donde se había conseguido un derecho del trabajo estable y seguro, ha ocurrido una radical y extrema comercialización del trabajo, que ha cerrado dicha temporada ${ }^{3}$.

La precarización estructural del trabajo ha conllevado varias consecuencias, como nuevas estratificaciones, el crecimiento de las desigualdades de renta, el empobrecimiento de amplias camadas de trabajadores, el aumento del ejército de trabajadores de reserva con la creación de una reserva de la reserva, la adaptación incondicionada de la vida de los trabajadores a las necesidades organizativas de las empresas privadas y de los organismos estatales, la alteración de los derechos sociales que han sido adaptados en función de los derechos de la empresa y del "crecimiento", el deterioro de la salud psicológica y física por la precariedad que erosiona. Los contratos de trabajo discontinuo, intermitente, compartido, arrendado, subcontratado, a llamada, y todas las otras formas de precariedad (encabezados por su prototipo: el trabajo irregular, y su extremo: el trabajo forzado), aplicadas primariamente al sector de la inmigración, han fragmentado y desconectado la mano de obra, facilitando la desvalorización general del trabajo y el desconocimiento de los trabajadores en el proceso de producción y reproducción de la vida social, hasta el punto de que, más que de re-comercialización del trabajo, se tendría que hablar de nulificación del trabajo y del trabajador.

Con la llegada de la crisis económica y la consiguiente radicalización de las políticas neoliberales, la precarización del trabajo se ha ampliado y profundizado aún más, ha dado un salto cualitativo y cuantitativo, y se ha ido más allá de la precariedad conocida en el pasado reciente. Hoy las fronteras de la precariedad 2.0 - voucher, vales de alimentación en lugar de un sueldo, prácticas, trabajo voluntario, gratuito, posted workers, socios de cooperativas ficticias - han ampliado sus fronteras, substituyendo parte del trabajo decente y parte de los propios trabajos precarios de "primera generación".

El proceso de precarización, tanto en su vieja versión como en la nueva, ha afectado la masa de trabajadores de maneras diferentes: los primeros y más

\footnotetext{
MAHMUD, Tayyab. Precarious existence and capitalism: a permanent state of exception.
} 
afectados han sido los inmigrantes, los jóvenes, las trabajadoras con hijos con bajo nivel de educación y cualificación, los older workers interesados por las reestructuraciones empresariales, los trabajadores poco cualificados, todos pertenecientes al segmento más inestable y vulnerable de la clase trabajadora europea. Este segmento, que no constituye una clase distinta ${ }^{4}$, separada, es parte estructural de la working class europea; esta underclass ha vivido un profundo proceso de empobrecimiento y marginalización y es destinataria constante de campañas racistas (en relación a su parte extranjera) o eslóganes nacionalistas y sirenas populistas (en relación a su parte local) ${ }^{5}$.

\section{La precarización de la inmigración en la Europa}

En la mayoría de Europa, la precarización estructural del trabajo ha interesado de manera importante a los inmigrantes, al menos por tres razones: 1) la combinación con políticas migratorias implementadas desde hace al menos veinte años por los países europeos; 2) el papel atribuido a la inmigración en los capitalismos europeos y el modo de funcionamiento del mercado del trabajo; 3) el irresistible avance del racismo anti-inmigrantes ocurrido en los primeros años del siglo XXI.

En muchos países europeos, los trabajadores inmigrantes son sujetos a una doble precarización, laboral y jurídica. La primera dimensión está incluida en la precarización del trabajo que acabamos de ver, mientras que la segunda está ligada a las políticas migratorias de diversos países que supeditan la permanencia legal en el territorio europeo a la existencia de un regular contrato de trabajo y enlazan los derechos sociales al estado migratorio, estratificándolos ${ }^{6}$. Estos principios han contribuido a la vuelta de la figura del Gastarbeiter ${ }^{7}$, pero en un contexto de capitalismo flexible con toda su fragmentación y polarización: la generalización del vínculo entre permiso de residencia y contrato de trabajo en una fase de crisis económica, elevado desempleo, precarización, es una espada de Damocles permanente sobre la cabeza de los inmigrantes, que los obliga a aceptar cualquier condición. La interacción entre estas dos dimensiones, en comparación con las décadas anteriores, ha producido una más profunda precarización de la condición de los inmigrantes que, divididos entre una condición de rigidez determinada por las leyes sobre inmigración y una condición de flexibilidad producida por las leyes sobre el trabajo, en

\footnotetext{
${ }^{4}$ Como opina STANDING, Guy. The Precariat.

5 ART, David. Inside the Radical Right; FLECKER, Jörg. Changing Working Life and the Appeal of the Extreme Right.

6 SCHIERUP, Carl-Ulrik et alii. Migration, Citizenship, and the European Welfare State; MORRIS Lydia, Managed Migration.

7 CASTLES, Stephen. Guestworkers in Europe: A Resurrection?; SAMERS, Michael. New Guest Worker Regimes?
} 
muchos países europeos se han encontrado en la situación de trabajadores huéspedes en un contexto de estancamiento económico, aumento de los contratos atípicos, debilitamiento del movimiento obrero y sindical. Por lo tanto, si por los años '60 y primeros '70 se puede hablar de una inserción laboral relativamente estable, en las décadas siguientes la realidad ha sido la de la precarización de la misma inserción laboral, y desde el punto de vista social, un refuerzo de los factores de exclusión o, por lo menos, de segregación.

Dentro de los capitalismos europeos se han subrayado las funciones de ejército de reserva y amortiguador social de la crisis históricamente atribuida a la inmigración ${ }^{8}$, así que los trabajadores inmigrantes han sido los primeros en probar el pasaje al trabajo precario y las formas de precariedad más extremas. Entretanto, los mecanismos de funcionamiento propios del mercado del trabajo - selección, concentración, especialización - han seguido canalizando la mayoría de los inmigrantes en los sectores y trabajos más precarios, más pesados, menos retribuidos, con el resultado de que en Europa su condición de trabajo - del acceso al trabajo al desempleo, de las tareas al sueldo, de la clasificación a la movilidad - presenta, si bien con formas y grados diferentes según el contexto, grandes desigualdades en comparación con los trabajadores nacionales. Se registran amplias áreas de desempleo, subempleo, sobreeducación, subclasificación laboral, precariedad contractual, en porcentaje más elevado y en el tiempo más prolongadas en comparación con la población mayoritaria, exacerbadas por la crisis ${ }^{9}$. Este empeoramiento está ligado en particular al pasaje de contratos a tiempo completo a contratos (formalmente) a tiempo parcial, al gran aumento de contratos temporarios y de trabajos intermitentes, a la caída o al riesgo de caída en el trabajo irregular o en el desempleo.

El avance del racismo institucional ha aumentado su precarización y ha reducido los derechos sociales. El ataque anti-inmigrantes en tema de legislación y de propaganda - funcional a explotar y controlar más profunda y sencillamente el trabajo inmigrante - ha soportado políticas migratorias caracterizadas por una mezcla de elementos de identidad y seguridad, con el fin de desincentivar el arraigo social y de incentivar migraciones circulares, temporarias, fluctuantes, según la necesidad de mano de obra just in time. La vuelta del asimilacionismo, tanto en políticas como en retóricas, ha conllevado

8 CASTLES, Stephen. Ethnicity and Globalization: From Migrant Worker to Transnational Citizen; SCHIERUP, Carl-Ulrik et alii. Migration, Precarity, and Global Governance: Challenges and Opportunities for Labour.

9 Entre los trabajadores inmigrantes se ha registrado un aumento en el desempleo (especialmente entre los hombres) y en el subempleo (producido por las disminuciones del horario de trabajo), una intensificación de la segregación profesional (en los sectores productivos y en las profesiones), la disminución de los sueldos. Véase OECD. International Migration Outlook 2013 (<http:// www.oecd-ilibrary.org/social-issues-migration-health/international-migration-outlook-2013 migr_outlook-2013-en); OECD. International Migration Outlook 2015. 
la máxima adaptación social de los inmigrados a las condiciones a las cuales están sometidos. Los medios de comunicación, al engendrar un régimen de representación racializado de la sociedad, orgánico a la inserción subordinada de los inmigrantes, han producido una diferenciación de las representaciones públicas de los inmigrantes funcional a su explotación diferencial, también debido a su precarización estratificada. Los eslóganes y discursos enfocados en la preferencia nacional ("La France d'abord", "Britain first", "prima gli italiani"), en una presencia necesariamente provisional, han respaldado la precarización reservada a la mayoría de los inmigrantes como fracción de clase racializada de la working class europea.

Al mismo tiempo, hay que subrayar el papel de la inmigración como vector de precarización. El mercado del trabajo es segmentado, pero la difusión de la precariedad entre los inmigrantes ha sido un factor de multiplicación y ampliación general de la precariedad que ha interesado una grande parte de los trabajadores; en este contexto, las políticas migratorias, por ejemplo, han tenido un valor general muy importante ya que han sentado las bases y anticipado políticas del trabajo muy similares ${ }^{10}$.

El proceso de convergencia a la baja de las políticas migratorias, empezado lentamente en los años '70 y '80, y que ha ganado fuerza en los años '90 y 2000, ha interesado la mayoría de los estados europeos y ha asistido a la confluencia de las distintas políticas migratorias en una única política migratoria de tipo selectivo, restrictivo y represivo ${ }^{11}$, que calibra la llegada de la inmigración según el aumento de flexibilidad necesario al mercado del trabajo. Este proceso ha sido acompañado por interminables campañas de denigración de los varios sectores de la inmigración (los indocumentados, los jóvenes de segunda generación, los barrios con grande presencia de inmigrantes), que han acabado afectando toda la inmigración e gran parte del mundo del trabajo, dividiéndolo a lo largo de múltiples líneas de diferenciación ${ }^{12}$.

En este contexto, en el que la inmigración es favorecida por el mercado y desfavorecida por las instituciones - según un juego de roles destinado a la explotación y a la opresión social y política de los inmigrantes - el verdadero objetivo de esa política migratoria a la baja no es tanto el bloqueo total de la inmigración o un cierre hermético de las fronteras, sino una inmigración instable, vulnerable, poco arraigada, con pocas demandas, y el empeoramiento de las condiciones de la migración, orgánico a la desvalorización general del trabajo también a través de la desvalorización de una parte. Históricamente, el

\footnotetext{
${ }^{10}$ BASSO, Pietro. Politiche migratorie e precarizzazione del lavoro.

${ }^{11}$ BASSO, Pietro (ed.). Razzismo di stato. Stati Uniti, Europa, Italia; MORICE, Alain, POTOT, Swanie (eds.). De l'ouvrier immigré au travailleur sans papiers.

12 Por ejemplo OUALI, Nouria. Le racisme au travail à Bruxelles et à Londres: un effet de la précarisation.
} 
uso de la mano de obra inmigrante en los países occidentales y a nivel mundial ha representado una de los instrumentos fundamentales para la desvalorización de la mano de obra en general, y ha constituido una reserva de mano de obra que ha permitido encarar las necesidades de la producción capitalista, bajando el coste del trabajo y ofreciendo una mano de obra súper-flexible obligada a una posición subordinada y de chantaje por las políticas migratorias y las discriminaciones institucionales ${ }^{13}$; hoy esta condición específica ha hecho de la inmigración un suelo donde el sistema de producción ha experimentado nuevas formas de división y organización del trabajo pensadas para incrementar la rentabilidad y la productividad, y un elemento de reestructuración y re-estratificación del mercado del trabajo a través de procesos que han llevado a la substitución de trabajo seguro por trabajo precario.

En este sentido políticas migratorias y políticas laborales cada vez más precarizadoras han sido complementares. Y la formación de una área de trabajo institucionalmente débil y precario, de una underclass de trabajadores temporarios que se substituyen el uno al otro en una rotación y circulación continuas, no solo ha permitido experimentar nuevas formas de explotación del trabajo a generalizar (ni de hacer una especie de deslocalización local), sino que también ha favorecido la nueva regulación global de las relaciones de trabajo marcados por la precariedad estable. La producción de indocumentados, por ejemplo, principales ejemplos de la precariedad total, forma parte integrante del proceso de precarización estructural del trabajo, mientras que la interminable campaña de denigración de estas personas afecta todo el sector de la inmigración (y del trabajo), culpando a ello por las medidas restrictivas y represivas relativas a toda la población europea que derivan, en realidad, de procesos que no dependen ni de la inmigración, ni, en general, del trabajo asalariado. Caso emblemático de estos procesos es Italia, que se ha transformado en una vanguardia de precarización del trabajo y de explotación de los inmigrantes.

\section{Italia, vanguardia de la precariedad laboral y de la explotación del trabajo inmigrante}

La frenética y constante actividad de reforma del mercado del trabajo que ha tenido lugar en Italia en las últimas dos décadas ha legalizado todas las formas de precarización del trabajo, a menudo experimentadas con antelación por los trabajadores inmigrantes. Esta actividad, desarrollada de manera integrada, ha interesado tanto la "flexibilidad de prestación" como la "flexibilidad de ocupación", a través de una larga serie de leyes y medidas de las cuales voy a citar solo las etapas principales: el acuerdo de julio 1993 entre Gobierno y

\footnotetext{
${ }^{13}$ POTTS, Lydia. The World Labour Market. A History of Migration.
} 
sindicatos, que ha abierto la puerta al trabajo a tiempo determinado y al trabajo ocasional; la ley 196/1997, que ha reglamentado el trabajo ocasional en forma de trabajo temporario; el Decreto Legislativo 368/2001, que ha liberalizado los contratos de duración determinada, con la posibilidad de repetirlos sin fin y que ha introducido otros tipos de "contratos atípicos"; la ley 30/2003, que ha ampliado desmesuradamente la precariedad a través de la abrogación de la prohibición de intermediación de mano de obra, el refuerzo de la suministración de trabajo y la previsión de más de veinte tipos de contratos de trabajo atípicos (del staff leasing al trabajo a llamada), en nombre de la contratación individual; la ley 148/2011, que ha socavado el contrato nacional de trabajo a través de la introducción de contratos de proximidad a nivel empresarial y territorial; la ley 92/2912, que ha ampliado y estabilizado la precariedad; el Jobs Act de 2014 ha institucionalizado la precariedad transformándola en regla, ya no excepción: ha eliminado el contrato de duración indeterminada, ha liberalizado el contrato a duración determinada haciéndolo a-causal, ha establecido un límite máximo de 5 renovaciones contractuales en 3 años (que no se aplican a la persona sino a la tarea y el empresario puede modificar la tarea y perpetuar la precariedad), ha liberalizado las tareas, ha abolido el artículo 18 del Estatuto de los Trabajadores, relativo a la protección de despedidas liberalizando las despedidas individuales y colectivas sin justa causa.

Las normas sobre inmigración han introducido y arraigado el vínculo entre permanencia, trabajo y hogar, institucionalizando el modelo de inserción de los inmigrantes basado en su clandestinización y precariedad social y laboral. Continuando la ley 39/1990, la ley 40/98 ha establecido por escrito las prácticas sociales existentes, confirmando que la condición de clandestinidad constituye para los trabajadores inmigrantes una etapa obligatoria y la regularización solo llega en un segundo momento (con subsanaciones o decreto flujos) según la voluntad discrecional de cada empresario. La ley 189/2002 - que concibe la permanencia como un elemento estrechamente vinculado al estado ocupacional, a la existencia de una relación de trabajo, único elemento de legitimación de la legalidad de la permanencia - ha redefinido de manera restrictiva los requisitos de la permanencia con la introducción del "contrato de permanencia", determinando una inmigración súper-chantajeable, colgando de un hilo, dispuesta a todo para no volver a la clandestinidad. Ha sometido la gran mayoría de los inmigrantes a una condición de extrema precariedad establecida por ley, aumentada por la interacción con las leyes laborales; la institución de un vínculo estrecho entre trabajo, permanencia y hogar, factor de producción institucional de clandestinidad, hace con que el inmigrante esté a la merced del empresario, único sujeto legitimado para pedir el permiso de trabajo para el inmigrante y con la facultad y el derecho subjetivo de pedir permiso de residencia. La ley 94/2009 ha precarizado aún más su condición 
introduciendo el permiso de residencia a puntos, articulado por créditos: como en una especie de Candyland, durante la temporada de validez del permiso de residencia el inmigrante obtiene o pierde puntos útiles para la renovación.

Por último, pero no menos importante, la fragmentación de los tipos de permiso de residencia y la pluralización de la duración de los permisos de residencia previstas por las normas sobre inmigración, combinadas con la multiplicidad de contratos atípicos previstos por la legislación laboral, han determinado una importante estratificación del estatuto jurídico y social de los inmigrantes, una pluralidad de categorías portadoras de condiciones y derechos diferenciados, de donde surge una jerarquía de la precariedad presente dentro del propio sector de la inmigración.

\subsection{Desigualdad laboral y pedagogía de la precariedad}

En los años Noventa la inserción laboral de los inmigrantes ocurrió en los trabajos y en las tareas peores de la franja secundaria del mercado del trabajo, sobretodo en sectores de gran intensidad de mano de obra y con mucho trabajo no declarado, que han usado estos trabajadores como factor de mantenimiento de elementos de explotación aguda ${ }^{14}$. En muchas áreas de agricultura, por ejemplo, el recurso a trabajadores inmigrantes ha consolidado un modelo laboral basado en el empleo sistemático de mano de obra precaria, irregular, mal pagada, llevando a la supervivencia del caporalato (explotación de jornaleros) bajo nuevas formas; sector de tránsito para miles de inmigrantes, ella ha convertido la rotación continua de trabajadores extranjeros sometidos a condiciones infernales en un pilar de su funcionamiento, poniendo entre la espada y la pared a la mano de obra local.

La transferencia hacia el sector industrial ocurrida en los años 2000 ha conllevado una mejora global en las condiciones de los inmigrantes, pero la inserción en el mercado del trabajo se ha caracterizado por una marcada canalización en las tareas menos cualificadas y con menor retribución, más peligrosas y nocivas. Ha crecido la participación al mercado del trabajo oficial, se ha verificado una mayor diversificación de los sectores de empleo, pero también se ha exacerbado la segmentación racial del mercado del trabajo, acompañada por situaciones de segregación laboral y racialización de los procesos productivos. El sistema productivo, caracterizado por unidades de dimensiones mediano-pequeñas, aprovechando de los segmentos más inestables o marginales del mercado del trabajo ha tenido a su disposición una reserva permanente de mano de obra barata para sustentar las nuevas formas de división y organización del trabajo. Las dinámicas propias del mercado del trabajo han hecho que la experiencia laboral de los inmigrantes

${ }_{14} \mathrm{MACIOTI}$ Immacolata, PUGLIESE, Enrico. L'esperienza migratoria. 
- en relación a colocación, tareas, clasificación, sueldo, accidentes, seguridad social - presente estructuralmente desigualdades marcadas en comparación con los trabajadores nacionales ${ }^{15}$. Entre los trabajadores inmigrantes (alrededor de 2.300 .000 unidades, cerca del $10 \%$ del empleo total) hay una marcada segregación laboral que los concentra en los trabajos manuales con bajas cualificaciones, reservando a la mayoría de ellos las tareas de obrero, albañil, trabajador agrícola, cuidador, criada, limpiadora, camarero ${ }^{16}$. Entre ellos se registran mayores tasas de desempleo $(16,2 \%$ vs $11,4 \%)$, de subempleo $(11,7 \%$ vs $4,2 \%$ ), de sobreeducación ( $40,9 \%$ vs $21,6 \%$ ), de precariedad contractual, de intermitencia laboral, que duran más tiempo. Estos elementos, juntados a una condición laboral que no mejora con la antigüedad de trabajo, a una gran presencia en trabajos con escasas posibilidades de carrera y bajos aumentos salariales relativos a la antigüedad, afecta los sueldos más bajos en comparación con los trabajadores nacionales (sueldo neto medio mensual $979 €$ vs $1.362 €)^{17}$. Esa desigualdad laboral, que se enlaza con desigualdades jurídicas, de vivienda, sanitaria y escolar que afectan a la mayoría de los inmigrantes ${ }^{18}$, es el motor permanente de la desigualdad racial que se ha desarrollado en Italia en las últimas décadas junto con las históricas desigualdades de clase, género y territorio existentes en el país.

Paralela e internamente a este modelo de inserción social hay la socialización de masa a la precariedad. Como la inserción laboral ocurre bajo las horcas caudinas del trabajo irregular y de la clandestinidad, antes de llegar a una situación de mínima estabilidad, los inmigrantes pasan una temporada, más o menos larga según las circunstancias, en la que ocurre la máxima explotación y una especie de "aclimatación" a la incertitud permanente y a la inferioridad social. Esta temporada, que afecta a los inmigrantes de manera diferenciada según el orden y el momento de llegada, según la consistencia y la organización del movimiento migratorio, los prepara, parafraseando y actualizando a Sayad, a un destino de jornaleros precarios a vida ${ }^{19}$. Estas "prácticas forzosas" de preparación a la precariedad permanente han interesado e interesan una amplia mayoría de los inmigrantes y es la piedra angular del camino de inserción social de la inmigración extranjera en Italia. Más: esta pedagogía de la precariedad es un pilar del modelo de dominación de la inmigración desarrollado en los años '80, reforzado en los años '90 e institucionalizado en los años 2000, que en términos de socialización se ha ampliado en una especie de efecto halo a

\footnotetext{
${ }^{15}$ CARITAS - MIGRANTES. Immigrazione. Dossier statistico 2012; FULLIN, Giovanna, REYNERI, Emilio. Low unemployment and bad jobs for new immigrants in Italy.

${ }^{16}$ En 2012 el 87,1\% trabajaba como "obrero y ocupaciones no cualificadas" (frente al 39,6\% de los locales). FONDAZIONE MORESSA. Rapporto annuale sull'economia dell'immigrazione 2012, p. 33.

17 UNAR. Dossier statistico immigrazione 2016.

18 SARACENO, Chiara et alii. Stranieri e disuguali.

${ }^{19}$ SAYAD, Abdelmalek. La double absence.
} 
otras franjas de trabajadores que inicialmente habían asistido a la precarización de los inmigrantes, creyendo que sólo fuese reservada a ellos (y de hecho durante una temporada fue así). La formación de un área de trabajo precario, vulnerable, compuesta por trabajadores inmigrantes y por algunas franjas marginales de trabajadores locales, ha contribuido a allanar el camino también hacia otras franjas de trabajadores, pero como veremos, la experimentación y la ampliación de la precariedad aún no han acabado.

\subsection{Nuevas formas de precariedad laboral}

Las nuevas fronteras del trabajo precario que interesan de manera relevante a los inmigrantes presentan un carácter extremo, moderno y de proximidad con la condición de algunas franjas de trabajadores nacionales. Entre ellas, voy a profundizar el trabajo pagado con voucher y el trabajo gratuito de los solicitantes de asilo.

El voucher, o bono de trabajo, es una forma de retribución del trabajo ocasional accesorio, que prevé una remuneración de $10 €$ por hora (de los cuales 70 céntimos como tasa de seguro, 1,30€ como cotización de pensión y 50 céntimos como contribución para la gestión del servicio de venta realizado por el estanco, oficina de correos o INPS [instituto de previsión social]). No es asimilable a un contrato de trabajo, así que no necesita de comunicación de contratación al Ministerio del Trabajo. El trabajador puede acumular cada año hasta 7.000€, de distintos empresarios, y puede recibir de cada empresario hasta 2.020€; el empresario no está sometido a ningún techo de gasto. El voucher no da derecho a reposo, vacaciones pagadas, prestaciones de enfermedad, permiso de maternidad o por matrimonio, subsidios familiares, indemnización por despido. Los ingresos resultantes de los voucher no deben ser declarados a Hacienda.

Inicialmente establecido para unas actividades ocasionales realizadas por categorías específicas (tutorías después de clases, pequeños trabajos realizados por estudiantes y jubilados), ha encontrado grande aplicación en los trabajos ocasionales previstos por la ley 30/2003 como trabajo doméstico, trabajo de cuidado, trabajos temporeros en la agricultura, caracterizados por una elevada presencia de inmigrantes. En 2008 su aplicación ha sido liberalizada en términos de sectores y categorías de trabajadores, en 2012 se ha eliminado la referencia a la actividad ocasional y accesoria, conectando el trabajo accesorio a la remuneración anual recibida por el trabajador, en 2014 ha subido el techo anual de remuneración de 5.000 a $7.000 €$, haciendo de los voucher una forma de trabajo y remuneración de masa: en 2015 se han vendido 115 millones de voucher, o sea 1,1 billones de euros ${ }^{20}$, un tercio usados en la construcción

${ }^{20}$ Fuente: BANCA DATI INPS. Disponible en: <http://www.inps.it/webidentity/ banchedatistatistiche/venduti/index.jsp > . Consultado: 20.09.3016. 
y mitad en el comercio, turismo y servicios (sectores con elevada presencia de inmigrantes); en los primeros diez meses de 2016 se han vendido 121,5 millones de voucher ${ }^{21}$. Hoy la situación afecta más de un millón y medio de trabajadores (hombres y mujeres), entre ellos estudiantes universitarios, jubilados, parados, trabajadores en movilidad. Entre los inmigrantes el aumento ha sido muy marcado: en 2008 los trabajadores extranjeros no comunitarios afectados fueron 174, mientras que en 2015 fueron 119.232, que en media en un año han recibido 66 bonos cada uno para una remuneración anual media neta de $497 €^{22}$.

En pocos años el voucher se ha transformado en un instrumento "universal", transversal a los sectores, usado para múltiples empleos y para actividades no estrictamente ocasionales, que ha parcialmente substituido los contratos precarios a duración determinada. De forma de remuneración se ha convertido en forma de empleo, pero en esa evolución ha dejado de existir la relación de trabajo, ha desaparecido, canalizando a los trabajadores en el más agudo subprecariado. Establecido para regular el trabajo accesorio, a menudo es usado de manera continuada como sustituto del trabajo semi-estable, sobretodo del contrato a tiempo parcial (voluntario o involuntario); también es muy usado como cobertura del trabajo totalmente irregular (en caso de controles o de accidente, al trabajador se le activa el bono just in time) o del trabajo parcialmente irregular (el trabajador es pagado parcialmente en negro y parcialmente por voucher). Introducido para combatir el trabajo irregular, el voucher lo facilita y reproduce, en lugar de eliminarlo.

El trabajo voluntario gratuito - actividad distinta del voluntariado y del trabajo comunitario - vivió una primera amplia experimentación en el sector de los refugiados y solicitantes de asilo ${ }^{23}$. En puro estilo workfare y "políticas activas del trabajo", la circular 14290/2014 del Ministerio del Interior establecía que las Prefetture celebrasen protocolos de acuerdo con las entidades locales (Ayuntamientos, Provincias, Regiones) y con las organizaciones del tercero sector para realizar proyectos de trabajo voluntario, realizados por solicitantes de asilo de forma voluntaria y gratuita, con finalidades sociales y no lucrativas, con cobertura de seguro. El objetivo de estos proyectos es triple: la superación de la "condición de actitud pasiva de los inmigrantes"; poner a su disposición

${ }^{21}$ Fuente: INPS. Osservatorio sul lavoro precario. Disponible en: <http:/www.inps.it/portale/ default.aspx?sID=\%3b0\%3b9899\%3b10156\%3b\&lastMenu=10156\&iMenu=1\&iNodo=1015 $6 \& \mathrm{p} 4=2>$.

22 FONDAZIONE MORESSA. Rapporto annuale sull'economia dell'immigrazione 2016, p. 70. Los sectores más interesados son el trabajo doméstico (29,5\%), jardinería y limpieza (15,4\%), turismo $(9,8 \%)$, servicios $(7,8 \%)$.

${ }^{23}$ Hago referencia a PASQUALETTO, Martina. II "volontariato" dei richiedenti asilo in Italia, Venecia: Università Ca' Foscari, Master sull'Immigrazione, Tesis, 2016. Véase también GJERGJI, Iside. Sulla governance delle migrazioni, p. 95-98. 
posibilidades de integración en el tejido social; mejorar el clima social sobre la temática de los refugiados para evitar una oposición popular contra ellos.

A finales de 2015 habían 91 protocolos (7 regionales, 14 provinciales, 70 municipales), que envolvían todo el territorio italiano y varios sujetos participantes: cooperativas sociales que se ocupan de acogida, diócesis, sindicatos, entidades públicas, hospitales, empresas con participación del Ayuntamiento. Los protocolos prevén, normalmente en la forma de prácticas voluntarias gratuitas, las siguientes actividades: limpieza de áreas públicas, pintura de bancos y cercas, recogida de hojas, mantenimiento de cementerios y jardines públicos.

Las razones y finalidades a la base de los distintos protocolos pueden ser resumidas en los siguientes puntos: integración social y conocimiento del contexto local por los solicitantes de asilo; la superación de la condición de actitud pasiva y de falta de laboriosidad presente en los centros de acogida y un uso productivo del tiempo; la educación de los solicitantes de asilo, invitados a adquirir una conciencia de la participación; la mejora de las competencias profesionales; la convivencia pacífica con la población local; la posibilidad de ser útiles para el territorio y agradecidos. Estas razones, bien resumidas en una entrevista reciente del Jefe de Departamento para las Libertades Civiles y la Inmigración del Ministerio del Interior ${ }^{24}$, incluyen elementos críticos porque: 1) identifica en la condición de prolongada falta de laboriosidad de los solicitantes de asilo - determinada en realidad por la naturaleza complicada y lenta de los procedimientos burocráticos - el detonante del descontento popular; 2) se atribuye la responsabilidad de dicha falta de laboriosidad a los solicitantes de asilo, públicamente representados como "refugiados vagos"; 3 ) se juzga que, para no gravar demasiado el presupuesto del estado, ellos deben ser útiles trabajando gratuitamente y demostrando que merecen lo que reciben; 4) se considera que los solicitantes de asilo sean por definición personas de educar. En la entrevista, el entrevistado añadió que para el futuro no se excluye una pequeña remuneración para servicios a tiempo completo en los "sectores que lo necesitan: agricultura, construcción, atención a las personas mayores (...) No pienso en una remuneración con tarifas nacionales, sino una remuneración que puede ser reducida: el recorte serviría para recuperar los costes de la acogida"; ni se excluye una vía rápida en el camino para obtener el estatuto de refugiado para los que participan a los proyectos: "Quien demuestra buena voluntad y capacidad de inserción en nuestro contexto social podría tener una atención diferente en la acogida (...). Hay el permiso humanitario. De momento solo se

\footnotetext{
${ }^{24}$ PICCOLILLO, Virginia. II Capo dell'Immigrazione Morcone. "Affidare ai profughi lavori utili". Corriere della Sera. 18.8.2016. Disponible en: <http://www.corriere.it/cronache/16_agosto_17/ capo-dell-immigrazione-morcone-affidare-profughi-lavori-utili-e732f624-64c4-11 e6-82810851fdf23454.shtml > . Consultado: 20.8.2016.
} 
da por razones de vulnerabilidad de niños y enfermos. Podríamos utilizarlo en este sentido. Después de un año la verificación serviría a incentivar conductas virtuosas". Esta doble propuesta implica al menos dos consecuencias: el pasaje del trabajo voluntario (concebido como indemnización al Estado) realizado en la prima etapa de acogida al trabajo mal pagado en sectores específicos labourintensive realizado en la segunda etapa de acogida; la alteración del derecho de asilo, considerado como una concesión que se obtiene demostrando buena voluntad y trabajando gratuitamente. En enero de 2017, el Gobierno ha presentado el nuevo plan sobre inmigración, que entre los puntos principales incluye el trabajo obligatorio para todos los solicitantes de asilo - lo que podría convertirse en requisito para la obtención de la protección internacional.

\section{Conclusión}

En primer lugar, la precariedad laboral que interesa los inmigrantes es parte del proceso global de precarización estructural del trabajo. Pero, si de un lado la precariedad laboral es una condición específica del trabajo inmigrante, del otro también es condición común de todo el trabajo asalariado, porque en el contexto de la economía de mercado capitalista la precariedad del trabajo es un dato estructural, constitutivo de las relaciones de trabajo, ha sido y sigue siendo la condición normal de los que viven de su trabajo.

En segundo lugar, hay que subrayar la conexión muy estrecha entre transformaciones del trabajo y alteraciones de los derechos sociales, entre precarización laboral y estratificación de los derechos sociales: los efectos de la flexibilidad organizacional y de la precariedad contractual no se han limitado a la súpersegmentación del mercado del trabajo y al crecimiento desproporcionado de los subocupados, se han ampliado al debilitamiento de los derechos laborales y a la restricción de la ciudadanía social. En el caso de los inmigrantes, la precariedad laboral representa un importante factor de exclusión o segregación porque a menudo el ejercicio de los derechos sociales es sometido al contrato de trabajo o al permiso de residencia. La vuelta del Gastarbeiter y la tendencia por los estados y la opinión pública hacia una migración temporal, han favorecido, en una temporada de precariedad estructural, una adquisición limitada y vinculada de derechos sociales, engendrando nuevas formas de desigualdad, un marco de derechos estratificados, una ciudadanía social de geometría variable, suportada por una xenofobia rampante en nombre del Estado del bienestar ${ }^{25}$. Sin embargo, el ataque a los derechos sociales de ciudadanía en marcha desde al menos dos décadas, una verdadera política de estado encargada de alterar su estructura y funciones, aunque haya empezado

\footnotetext{
${ }^{25}$ BURNETT, Jon. The war on welfare and the war on asylum, p. 96-100; SPIRE, Alexis. Xénophobes au nom de I'Etat social. Le Monde diplomatique, dec. 2013. Disponible en: <http://www. monde-diplomatique.fr/2013/12/SPIRE/49901 > . Consultado: 22.03.2014.
} 
del sector de la inmigración, no se ha limitado a este sector, sino que se ha ampliado a otras franjas de la población, y por eso hay que empezar a considerar la idea de una ciudadanía global capaz de contener el deslizamiento hacia abajo y elevar el estándar mínimo.

\section{Bibliografía}

ANTUNES, Ricardo. The Meanings of Work: Essay on the Affirmation and Negation of Work. Leiden/Boston: Brill, 2013.

ART, David. Inside the Radical Right. Cambridge: Cambridge University Press, 2011.

BASSO, Pietro. Politiche migratorie e precarizzazione del lavoro. In COIN, Francesca (ed.). Gli immigrati, il lavoro, la casa. Milán: Franco Angeli, 2004, p. 82-117.

BASSO, Pietro (ed.). Razzismo di stato. Stati Uniti, Europa, Italia. Milán: Franco Angeli, 2010.

BURNETT, Jon. The war on welfare and the war on asylum. Race and Class, v. 57, n. 2, 2015, p. 96-100.

CARITAS - MIGRANTES. Immigrazione. Dossier statistico 2012. Roma: Idos, 2012.

CASTLES, Stephen. Ethnicity and Globalization: From Migrant Worker to Transnational Citizen. Londres: Sage, 2000.

CASTLES, Stephen. Guestworkers in Europe: A Resurrection?. International Migration Review, v. 40, n. 4, 2006, p. 741-766.

DOOGAN, Kevin. New capitalism? The transformation of work. Cambridge, Malden: Polity Press, 2009.

FLECKER, Jörg. Changing Working Life and the Appeal of the Extreme Right. Aldershot: Ashgate, 2007.

FONDAZIONE MORESSA. Rapporto annuale sull'economia dell'immigrazione 2016. Boloña: Il Mulino, 2016.

FULLIN, Giovanna; REYNERI, Emilio. Low unemployment and bad jobs for new immigrants in Italy. International Migration, v. 49, n. 1, 2011, p. 118-147.

GJERGJI, Iside. Sulla governance delle migrazioni. Milán: Franco Angeli, 2016.

HEAD, Simon. The New Ruthless Economy: Work and Power in the Digital Age. New York: Oxford University Press, 2003.

HUWS, Ursula. Labor in the Global Digital Economy. Nueva York: Monthly Review Press, 2014.

JÜRGENS, Ulrich; KRZYWDZINSKI, Martin. New Worlds of Work. Oxford: Oxford University Press, 2016.

MACIOTI, Immacolata; PUGLIESE, Enrico. L'esperienza migratoria. Roma-Bari: Laterza, 2003.

MAHMUD, Tayyab. Precarious existence and capitalism: a permanent state of exception. Southwestern Law Review, n. 44, 2015, p. 699-726.

MORICE, Alain; POTOT, Swanie (eds.). De l'ouvrier immigré au travailleur sans papiers. Les étrangers dans la modernisation du salariat. Paris: Karthala, 2010. 
MORRIS, Lydia. Managed Migration. Civic Stratification and Migrants Rights. Londres: Routledge, 2002.

OECD. International Migration Outlook 2015. Disponible en: <http://www.oecdilibrary.org/social-issues-migration-health/international-migration-outlook-2015 migr_outlook-2015-en>. Consultado: 22.01.2016.

OUALI, Nouria. Le racisme au travail à Bruxelles et à Londres: un effet de la précarisation. Les Mondes du Travail, n. 7, 2009, p. 87-101.

POTTS, Lydia. The World Labour Market. A History of Migration. Londres: Zed Books, 1990.

SAMERS, Michael. New Guest Worker Regimes? In AMELINA, Anna et alii. An Anthology of Migration and Social Transformation. Cham: Springer, 2015, p. 121-134.

SARACENO, Chiara et alii. Stranieri e disuguali. Boloña: II Mulino, 2013.

SAYAD, Abdelmalek. La double absence. Paris: Seuil, 1999.

SCHIERUP, Carl-Ulrik et alii. Migration, Citizenship, and the European Welfare State. Oxford: Oxford University Press, 2006.

SCHIERUP, Carl-Ulrik et alii. Migration, Precarity, and Global Governance: Challenges and Opportunities for Labour. Oxford: Oxford University Press, 2015.

STANDING, Guy. The Precariat. Londres-Nueva York: Bloomsbury, 2011.

UNAR. Dossier statistico immigrazione 2016. Roma: Idos, 2016.

Recibido para publicación en 24.01.2017

Aceptado para publicación en 06.03.2017

Received for publication in January 24, 2017

Accepted for publication in March 06 ${ }^{\text {th }}, 2017$

ISSN impresso 1980-8585

ISSN eletrônico 2237-9843

http://dx.doi.org/10.1590/1980-85852503880004905 